\title{
COX-2 structural analysis and docking studies with gallic acid structural analogues
}

\author{
M Amaravani, Nirmal K Prasad and Vadde Ramakrishna*
}

\begin{abstract}
Emblica officinalis is an ayurvedic herbal plant. The compounds isolated from this plant have good inhibitory effects against cyclooxygenase-2 (COX-2), among them gallic acid (GA) has the highest inhibitory effect. COX-2 (1.14.99.1) is an oxidoreductase having a role in prostaglandin biosynthesis, inflammatory responses and in cardiovascular events. COX-2 has gained special focus on research since past few decades. The sequence and structural studies reveals Mus musculus COX-2 shares the common conserved sequence and structural pattern with human COX-2. Molecular modeling and docking analysis with gallic acid and their structural analogues showed that 2-[(2E,4E)hexa-2,4-dienyl]-3,4,5-trihydroxybenzoic acid, (3,4,5-trihydroxybenzoyl) 3,4,5-trihydroxybenzoate and 3-hydroxy-4sulfooxybenzoic acid are more interactive and binding strongly than gallic acid at active site. Hence these three compounds should be considered as strong inhibitors for COX-2.
\end{abstract}

Keywords: Cyclooxygenase, COX-2, Gallic acid, Indian gooseberry, Docking studies

\section{Introduction}

COX-1 and COX-2 are two distinct isoforms of cyclooxygenase, and plays a vital role in conversion of arachidonic acid to prostaglandins (Lipsky et al. 1998; Vane et al. 1998). Prostaglandins (PGs) are involved in various pathophysiological processes like inflammatory responses, carcinogenesis and in cardiovascular events. COX-2 is not detectible in most normal tissues, but is induced by proinflammatory cytokines, growth factors and carcinogens, implying a role for COX-2 in both inflammation and control of cell growth (Subbaramaiah et al. 1996). In inflammatory tissues such as rheumatoidal synovium expression of COX-2 is up regulated and produce prostaglandin precursors which ultimately converted in to prostaglandins (Prasit et al. 1999). The recent studies on selective inhibition of COX-2 caused suppression of inflammation and azoxymethane-induced colon cancer have shown the importance of COX-2 as a target for anti-inflammatory and anticancer therapy (Dannhardt and Kiefer, 2001; Subhashini et al. 2004; Amaravani et al. 2006). Taken together, these data strongly suggest that suppressing levels of COX-2 will be an effective strategy for inhibiting inflammation and carcinogenesis.

\footnotetext{
* Correspondence: vrkrishna70@gmail.com

Department of Biotechnology \& Bioinformatics, Yogi Vemana University, Kadapa 516 003, A,P. INDIA
}

Non-steroidal anti-inflammatory drugs (NSAIDs) are effective against inflammation and are observed to inhibit PG biosynthesis. NSAIDs inhibit both isoforms of cyclooxygenases (COX), but they are also associated with well-known side effects such as gastrointestinal side effects and renal function suppression (Herschman, 1996). It is known that selective COX-2 inhibitors can provide anti-inflammatory agents devoid of the undesirable effects associated with classical non-selective NSAIDs (DeWitt, 1999). As a consequence, increasing interest has been devoted to the synthesis of inhibitors of COX-2 by means of modification of well-known non-selective agents. Apart from selective and nonselective inhibitors, many natural products have also been identified as COX-2 inhibitors (Zhang et al. 1999). As part of the search for natural anti-inflammatory agents from medicinal plants, Emblica officinalis extracts showed good medicinal values towards inflammation. Gallic acid (GA) is a naturally occurring polyhydroxyphenolic compound and an excellent free radical scavenger to inhibit COX isoforms (Madlener et al. 2007; Pal et al. 2010; Reddy et al. 2010). Presence of high levels of gallic acid in Emblica officinalis gives a special status and medicinal value for treating inflammatory diseases (Ramakrishna et al. 2011).

\section{Springer}

(c) 2012 Amaravani et al.; licensee Springer. This is an Open Access article distributed under the terms of the Creative Commons Attribution License (http://creativecommons.org/licenses/by/2.0), which permits unrestricted use, distribution, and reproduction in any medium, provided the original work is properly cited. 


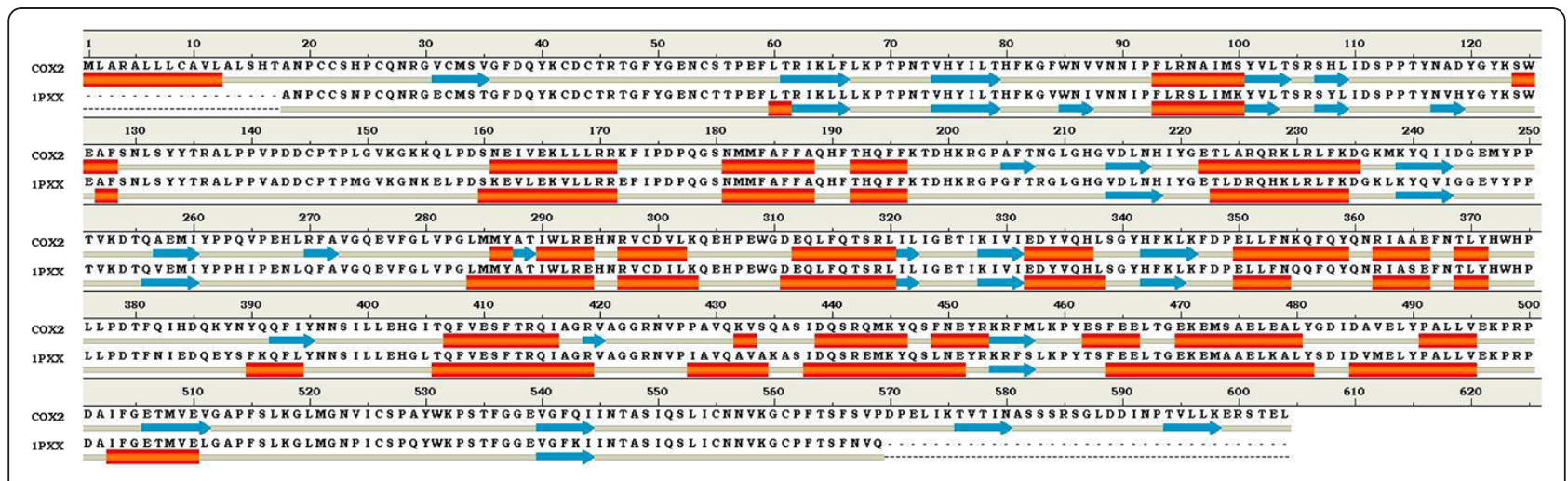

Figure 1 Secondary structural comparison of human COX-2 and template.

The present work focuses on the structural analysis of COX-2, interaction studies with gallic acid at active site and screening of gallic acid structural analogues. COX-2 active site analysis and molecular docking analysis enabled us to find better inhibitors as compared to gallic acid. These interaction studies are very useful to understand the mechanism of COX-2 catalyzed enzymatic reactions as well as the role of bioactive compounds interaction with active site residues. The approach is applicable in engineering 3D structures of enzymatic models, and studying interactions of active site residues with ligands (Nirmal et al. 2011a).

\section{Material and methods}

\section{Secondary structural analysis}

Human COX-2 protein and its structural homologue protein sequences were retrieved from the NCBI protein database (www.ncbi.nlm.nih.gov). Pair wise sequence alignment of sequences was generated by Clustal W 2.0 (http:// www.ebi.ac.uk/Tools/clustalw2/index.html) and analyzed to map the secondary structural conservation and variations. Secondary structural analysis was carried out by using Bioedit 7.0 (Hall, 1999) and Discovery Studio Viewer (www.accelrys.com).

\section{COX-2 Homology Modeling and optimization}

To build the COX-2 homology model, a BLASTp algorithm against Protein Data Bank (PDB) was used to carry out the sequence homology searches. Crystal structure of Mus musculus cyclooxygenase 2 (PDB ID: 1PXX) was taken as a template to build homology model. The Modeller 9v7 program (Sali and Blundell, 1993) was employed to generate the $3 \mathrm{D}$ models of COX-2. The model with high score was validated by the Procheck (Laskowski et al. 1993), VADAR (Willard et al. 2003) and ProSA (Wiederstein and Sippl, 2007). Further the model was refined by energy minimization. The energy minimization was performed using the NAMD package (Phillips et al. 2005). The optimized model was subjected to quality assessment with respect to its geometry and energy and then subjected to molecular docking. Ramachandran plot was utilized for geometric evaluation. ProSA program was employed to evaluate the quality of model and examine the energy of residue-residue interactions using a distance-based pair potential. The gallic
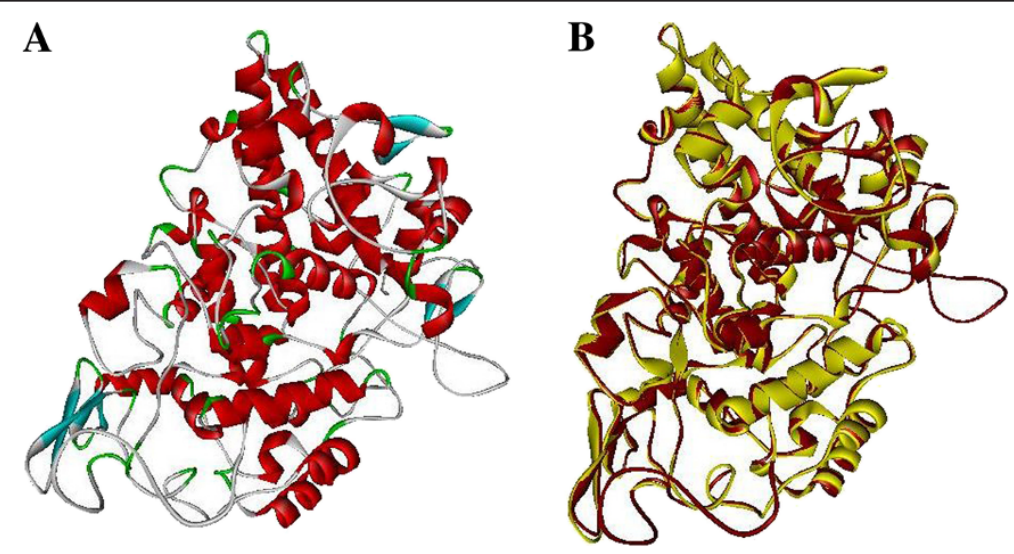

Figure 2 Final 3- D model of COX-2 (A) and superimposition with the template (B). 


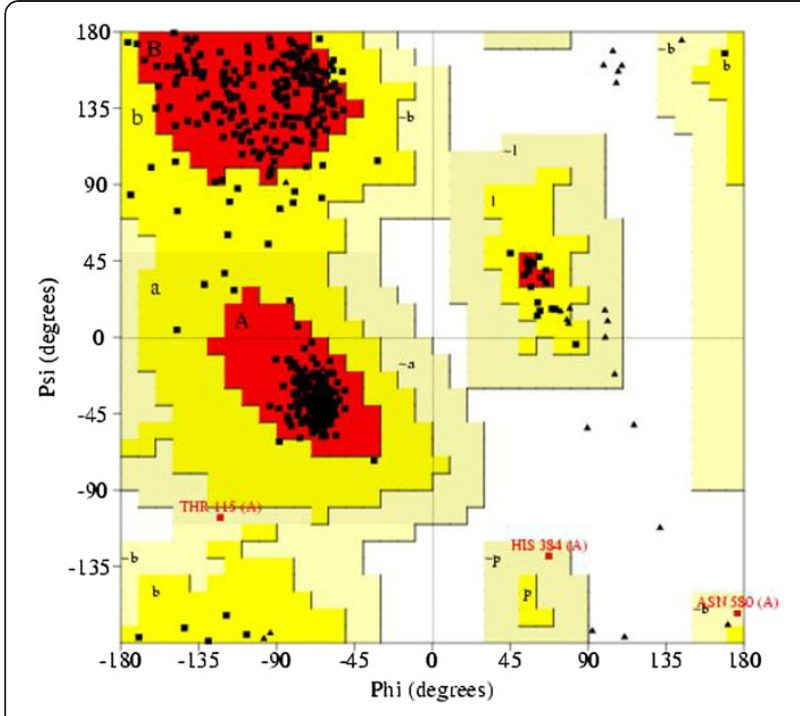

Figure 3 COX-2 homology model Ramachandran plot.

acid and its structural analogue molecules downloaded from Pubchem database of NCBI (Wang et al. 2009), and converted to 3D structure with VEGA ZZ software (Pedretti et al. 2004). These molecules were geometrically optimized for further use in docking. $\mathrm{C}$ alpha and back bone atoms root mean square deviation (RMSD) of template and COX-2 model was calculated by magic fit program (Guex and Peitsch, 1997).

Model energy minimization and molecular dynamics $3 \mathrm{D}$ structure refinement of COX-2 was carried out using energy minimization and molecular dynamics. It was

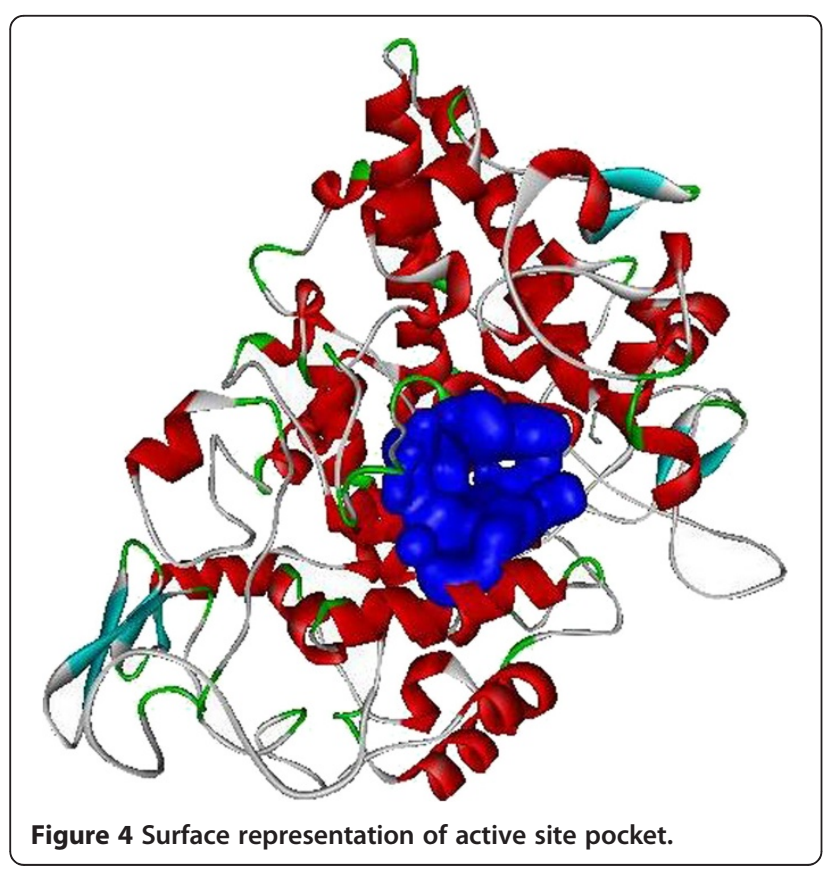

performed using Nano Molecular Dynamics (NAMD 2.6). The simulations and energy minimization were carried out in 50,000 step minimization of the designed side chains and solvent to remove bad contacts. Minimum switching distance of $8.0 \AA$ and a cut off of $12.0 \AA$ for Vander Walls interactions was used, pair list of the nonbonded interactions was recalculated every 20 steps with a pair list distance of $13.5 \AA$. The resultant energy minimized protein models were used for the active site identification and for docking with substrates.

\section{Active site analysis}

The substrate accessible pockets and active sites of COX2 were identified by computed atlas of surface topography of proteins (CASTp) calculation (Dundas et al. 2006) and CCDC GOLD (Jones et al. 1997; Verdonk et al. 2003). To test the accessibility of the pockets were tested by docking with randomly selected inhibitor molecules. The identified pockets were analyzed for amino acid cluster groups based on the solvent exposed active site atoms and bonding capacity of the polar groups.

\section{Docking analysis and inhibitor screening}

Gallic acid and its structural analogues are obtained from Pubchem database of NCBI and converted into 3D structures with VEGA ZZ software. The docking was carried out at the binding sites by CCDC's GOLD (genetic optimization for ligand docking). One-hundred genetic algorithm (GA) runs were performed for each compound, and 10 ligand bumps were allowed in an attempt to account for mutual ligand/target fit. The binding region for the docking study was defined as a $10 \AA$ radius sphere centered on the active site. For each of the GA run a maximum number of 100,000 operations were performed on a population of 100 individuals with a selection pressure of 1.1. The number of islands was set to 5 with a niche size of 2 . The weights of crossover, mutation, and migration were set to 95,95 , and 10 respectively. The scoring function Gold Score implemented in GOLD was used to rank the docking positions of the molecules, which were clustered together when differing by more than $2 \AA$ RMSD (Phogat et al. 2010; Nirmal et al. 2011b). The best ranking clusters for each of the molecules were selected. Hydrogen bonds, bond lengths and close contacts between enzyme active site and ligand atoms were analyzed.

\section{Results and discussion}

\section{Secondary structural features}

Comparative secondary structural analysis of COX-2 with template reveals that the secondary structural elements were well conserved. The secondary structural comparison of COX-2 was presented in Figure 1. Secondary structure of the COX-2 showing same pattern 
as compared to template secondary structure except few small stretches of beta sheets ( 2 to 3 amino acids) but this can be ignored.

\section{COX-2 model}

The COX-2 is a 604 amino acids protein. Crystal structures of COX-2 from different species have already been determined and available in PDB. Among them, Mus musculus cyclooxigenase 2 (PDB ID: 1PXX) showed the highest sequence identity (87\%) with COX-2. Practically, at this level of sequence identity, it is good enough to use $1 \mathrm{PXX}$ as a template, in order to obtain high quality alignment for the structure prediction by homology modeling. COX-2 homology (A) and superimposed pose with Template (B) was shown in Figure 2. The geometry of the final model of COX-2 was evaluated with Ramachandran's plot calculations computed with the PROCHECK program. This result revealed $91.8 \%$ of the residues were in the core region, $7.6 \%$ residues in the allowed regions and $0.6 \%$ in generously allowed region. COX-2 Ramachandran plot was depicted in Figure 3. The PROSA analysis of the model showed maximum residues to have negative interaction energy with very few residues displayed positive interaction energy and the overall interaction energy of the model was -7.69 $\mathrm{kcal} / \mathrm{mol}$, which is quite similar to the template $\mathrm{Z}$ score.

$\mathrm{C} \alpha$ atoms and back bone atoms RMSD of the model and template was $0.35 \AA$. The mean residue volume and total packing volume of the model are $153.9 \AA^{3}$ and $92962.6 \AA^{3}$ respectively. VADAR analysis of the model showed, the mean helix phi, psi and omega angles are $-65.1,-40.4$ and -178.3 respectively, which is promising residue packing when compared to the crystal, structure information. Hence, the final model which proved to be well validated in terms of geometry and energy profiles suggests that the model is good enough to be an initial point for our next stage of molecular docking studies.

\section{Active site composition}

After the final homology model was built, the possible ligand-binding site of COX-2 was searched by CASTp calculation and CCDC GOLD. Non-steroidal anti-inflammatory drug (NSAID) binding site was selected
Table 1 Properties of COX-2 active site residue composition and accessible atoms

\begin{tabular}{|c|c|c|c|}
\hline S.No & Residue & No. of Hydrogen donors & Atoms \\
\hline 1 & ALA185 & - & - \\
\hline 2 & PHE186 & - & - \\
\hline 3 & PHE187 & - & - \\
\hline 4 & ALA188 & - & - \\
\hline 5 & GLN189 & - & - \\
\hline 6 & HIS190 & - & - \\
\hline 7 & THR192 & 1 & HG1 \\
\hline 8 & HIS193 & - & - \\
\hline 9 & GLN194 & - & - \\
\hline 10 & PHE196 & - & - \\
\hline 11 & THR198 & - & - \\
\hline 12 & ASN368 & 1 & $2 \mathrm{HD} 2$ \\
\hline 13 & LEU370 & - & - \\
\hline 14 & TYR371 & - & - \\
\hline 15 & HIS372 & - & - \\
\hline 16 & TRP373 & 1 & $\mathrm{H}$ \\
\hline 17 & HIS374 & 1 & HE2 \\
\hline 18 & LEU376 & - & - \\
\hline 19 & LEU377 & - & - \\
\hline 20 & VAL433 & - & - \\
\hline 21 & SER437 & - & - \\
\hline 22 & GLN440 & 1 & 1HE2 \\
\hline 23 & TYR490 & - & - \\
\hline 24 & LEU493 & - & - \\
\hline 25 & LEU494 & - & - \\
\hline
\end{tabular}

for docking studies. The volume and area of active site are $5331.2 \AA^{3}$ and $1651.6 \AA^{2}$ respectively. The active site accommodate by 25 amino acids i.e., ALA185, PHE186, PHE187, ALA188, GLN189, HIS190, THR192, HIS193, GLN194, PHE196, THR198, ASN368, LEU370, TYR371, HIS372, TRP373, HIS374, LEU376, LEU377, VAL433, SER437, GLN440, TYR490, LEU493 and LEU494. There were 5 hydrogen donor groups present in the active site. COX-2 active site was shown in Figure 4. The comparison of the overall folding and the structure of active site between COX-2 and the template protein reveal a high
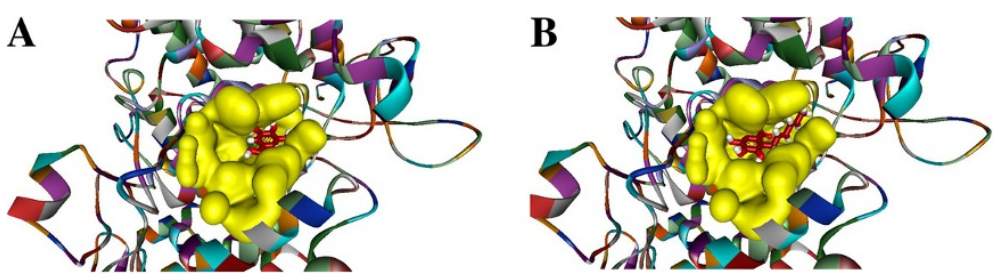

Figure 5 Docked conformations of (A) Gallic acid and (B) 2-[(2E,4E)-hexa-2,4-dienyl]-3,4,5-trihydroxybenzoic acid in the active site pocket. 
structural homology. Active site composition features were depicted in Table 1.

\section{COX-2 interaction analysis with inhibitors}

Initial screening of gallic acid structural analogues was done by CCDC GOLD docking. There were 59 gallic acid structural analogues are screened. All the screened gallic structural analogues were accessibleand downloaded from the library (www.ioib.in/products/GASAL). This initial screening studies revealed 2-[(2E,4E)-hexa-2,4dienyl]-3,4,5-trihydroxybenzoic acid, (3,4,5-trihydroxybenzoyl) 3,4,5-trihydroxybenzoate, 3-hydroxy-4-sulfooxybenzoic

Table 2 Docking statistics

\begin{tabular}{|c|c|c|c|c|}
\hline S.No & Ligand & Gold score & $\mathrm{H}$ bond atoms & H Bond length $(\AA)$ \\
\hline \multirow[t]{2}{*}{1} & 3,4,5-trihydroxybenzoic acid (Gallic acid) & 28.2848 & ALA185:O-H15 & 1.745 \\
\hline & & & ALA188:O-H16 & 2.692 \\
\hline \multirow[t]{2}{*}{2} & 2-[(2E,4E)-hexa-2,4-dienyl]-3,4,5-trihydroxybenzoic acid & 45.4076 & THR192:HG1-O3 & 2.099 \\
\hline & & & HIS372:ND1-H24 & 1.832 \\
\hline \multirow[t]{5}{*}{3} & (3,4,5-trihydroxybenzoyl) 3,4,5-trihydroxybenzoate & 42.7486 & ALA185:O-H32 & 1.441 \\
\hline & & & THR192:HG1-O5 & 1.363 \\
\hline & & & ASN368:1HD2-O7 & 2.161 \\
\hline & & & ASN368:2HD2-O3 & 2.071 \\
\hline & & & HIS374:H-O8 & 2.298 \\
\hline \multirow[t]{5}{*}{4} & 3-hydroxy-4-sulfooxybenzoic acid & 41.8640 & THR192:HG1-O7 & 2.448 \\
\hline & & & ASN368:O-H2O & 1.942 \\
\hline & & & ASN368:2HD2-O6 & 1.849 \\
\hline & & & $\mathrm{HIS372:2ND1-H19}$ & 2.399 \\
\hline & & & TRP373:O-H21 & 2.561 \\
\hline \multirow[t]{6}{*}{5} & 3,4-dihydroxy-2-sulfooxybenzoic acid & 40.5943 & THR192:HG1-O3 & 2.407 \\
\hline & & & ASN368:O-O4 & 2.410 \\
\hline & & & ASN368:1HD2-O4 & 2.525 \\
\hline & & & TYR371:O-H19 & 2.219 \\
\hline & & & THR373:H-O2 & 2.456 \\
\hline & & & THR373:H-O5 & 2.271 \\
\hline \multirow[t]{2}{*}{6} & prop-2-enyl 3,4,5-trihydroxybenzoate & 40.2194 & THR192:HG1-O2 & 2.127 \\
\hline & & & HIS372:ND1-H21 & 1.586 \\
\hline \multirow[t]{4}{*}{7} & 4-hydroxybutyl 3,4,5-trihydroxybenzoate & 39.9954 & THR192:OG1-H29 & 1.839 \\
\hline & & & THR192:HG1-O3 & 2.322 \\
\hline & & & THR192:HG1-06 & 2.009 \\
\hline & & & ASN368:2HD2-O4 & 2.546 \\
\hline \multirow[t]{3}{*}{8} & 3-hydroxypropyl 3,4,5-trihydroxybenzoate & 39.9464 & ALA185:O-H28 & 1.954 \\
\hline & & & THR192:OG1-H25 & 2.275 \\
\hline & & & THR192:HG1-O2 & 2.305 \\
\hline \multirow[t]{5}{*}{9} & bis(3,4,5-trihydroxyphenyl)methanone & 39.0007 & ALA185:O-H30 & 1.847 \\
\hline & & & THR192:OG1-H25 & 2.124 \\
\hline & & & THR192:HG1-O2 & 2.181 \\
\hline & & & ASN368:O-H27 & 1.490 \\
\hline & & & ASN368:1HD2-O6 & 2.024 \\
\hline \multirow[t]{3}{*}{10} & 1-(3,4,5-trihydroxyphenyl)pentan-1-one & 38.8825 & TRP192:HG1-O2 & 1.995 \\
\hline & & & HIS372:ND1-H28 & 1.451 \\
\hline & & & TRP373:H-O1 & 2.563 \\
\hline
\end{tabular}


acid, 3,4-dihydroxy-2-sulfooxybenzoic acid, prop-2-enyl 3,4,5-trihydroxybenzoate, 4-hydroxybutyl 3,4,5-trihydroxybenzoate, 3-hydroxypropyl 3,4,5-trihydroxybenzoate, bis (3,4,5-trihydroxyphenyl)methanone and 1-(3,4,5-trihydroxyphenyl)pentan-1-one molecules having high affinity at active site and binding firmly. Further docking analysis of the screened inhibitors revealed 2-[(2E,4E)-hexa-2,4dienyl]-3,4,5-trihydroxybenzoic acid, (3,4,5-trihydroxybenzoyl) 3,4,5-trihydroxybenzoate and 3-hydroxy-4sulfooxybenzoic acid are producing high Gold fitness score which shows high binding affinity at active site. The docking conformations of COX-2 with screened inhibitors were shown in Figure 5. The Gold Score of all interactions reveals that, among all the ligands, 2[(2E,4E)-hexa-2,4-dienyl]-3,4,5-trihydroxybenzoic acid exhibits the highest fitness score of 45.40. COX-2 docking statistics were depicted in Table 2.

\section{Conclusion}

COX-2 plays a prime role in the prostaglandins biosynthesis pathway as it provides prostaglandin $\mathrm{H}_{2}$, which is precursor for the formation of all other prostaglandins. Homology model of COX-2 showed $91.8 \%$ of the residues were in the core region, $7.6 \%$ residues in the allowed regions and $0.6 \%$ in generously allowed region of Ramchandran plot, suggesting the modeled COX-2 structure was reliable for the docking studies. The active site analysis showed 25 residues are present at surface accessible region of COX-2 active site. Top ten ranked gallic acid structural analogues on docking reveals that the 2-[(2E,4E)-hexa-2,4dienyl]-3,4,5-trihydroxybenzoic acid has more affinity at active site than others. This information has potential implications to understand the mechanism of COX-2 related enzymatic inhibition reactions, and also applicable in the prediction of more effective inhibitors and engineering 3D structures of other enzymes as well.

\section{Competing interests}

The authors declare that they have no competing interests.

\section{Authors' contributions}

MA carried out the sequence alignment, homology and modeling and helped in docking. NKP carried out the Docking interaction Studies and drafted the manuscript. VR planned the work, drafted the manuscript and supervised the entire work. All authors read and approved the final manuscript.

\section{Acknowledgements \\ V. Ramakrishna thankful to the Agri Science Park, A.P., India for supporting} this research work.

Received: 1 August 2012 Accepted: 29 November 2012 Published: 10 December 2012

\section{References}

Amaravani M, Reddy RN, Reddy GV, Reddanna P, Reddy MR (2006) A comparison of computer aided drug design methods for calculating relative binding affinities of COX-2 inhibitors. Indian J Chem 45A:174-181

Dannhardt G, Kiefer W (2001) Cylcooxygenase inhibitors - current status and future prospects. Eur J Med Chem 36:109-126
DeWitt DL (1999) Cox-2-Selective Inhibitors: The New Super Aspirins. Mol Pharmacol 55:625-631

Dundas J, Ouyang Z, Tseng J, Binkowski A, Turpaz Y, Liang J (2006) CASTp: computed atlas of surface topography of proteins with structural and topographical mapping of functionally annotated residues. Nucleic Acids Res 34:116-118

Guex N, Peitsch MC (1997) SWISS-MODEL and the Swiss-PdbViewer: an environment for comparative protein modeling. Electrophoresis 18:2714-2723

Hall TA (1999) BioEdit: a user-friendly biological sequence alignment editor and analysis program for Windows 95/98/NT. Nucl Acids Symp Ser 41:95-98

Herschman HR (1996) Prostaglandin synthase 2. Biochim Biophys Acta 1299:125-140

Jones G, Willett P, Glen RC, Leach AR, Taylor R (1997) Development and Validation of a Genetic Algorithm for Flexible Docking. J Mol Biol 267:727-748

Laskowski RA, Macarthur MW, Moss DS, Thornton JM (1993) Procheck: a program to check the stereochemical quality of protein structures. J Appl Crystallogr 26:283-291

Lipsky PE, Abramson SB, Crofford L, DuBois RN, Simon L, van de Putte LBA (1998) The classification of cyclooxygenase inhibitors. J Rheumatol 25:2298-2303

Madlener S, IIImer C, Horvath Z, Saiko P, Losert A, Herbacek I, Grusch M, Elford HL, Krupitza G, Bernhaus A, Fritzer-Szekeres M, Szekeres T (2007) Gallic acid inhibits ribonucleotide reductase and cyclooxygenases in human HL-60 promyelocytic leukemia cells. Cancer Lett 245:156-162

Nirmal Parasad K, Vindal V, Siva LN, Ramakrishna V, Kunal SP, Srinivas M (2011) In silico analysis of Pycnoporus cinnabarinus laccase active site with toxic industrial dyes. J Mol Model. doi:10.1007/s00894-011-1215-0

Nirmal Prasad K, Vindal V, Kumar V, Kabra A, Phogat N, Kumar M (2011) Structural and docking studies of Leucaena leucocephala Cinnamoyl CoA reductase. J Mol Model 17:533-541

Pal C, Bindu S, Dey S, Alam A, Manish Goyal M, labal S, Maity P, Adhikari SS, Bandyopadhyay U (2010) Gallic acid prevents nonsteroidal anti-inflammatory drug-induced gastropathy in rat by blocking oxidative stress and apoptosis. Free Radic Biol Med 49(2010):258-267

Pedretti A, Villa L, Vistoli G (2004) VEGA - An open platform to develop chemo-bioinformatics applications, using plug-in architecture and script" programming. J Comput Aided Mater Des 18:167-173

Phillips JC, Braun R, Wang W, Gumbart J, Tajkhorshid E, Villa E, Chipot C, Skeel RD, Kale L, Schulten K (2005) Scalable molecular dynamics with NAMD. J Comput Chem 26:1781-1802

Phogat N, Vindal V, Kumar V, Inampudi K, Prasad NK (2010) Sequence analysis, in silico modeling and docking studies ofCaffeoyl CoA-O-methyltransferase of Populus trichopora. J Mol Model 16:1461-1471

Prasit P, Wang Z, Brideau C, Chan CC, Charleson S, Cromlish W, Ethier D, Evans JF, Ford-Hutchinson AW, Gauthier JY, Gordon R, Guay J, Gresser M, Kargman S, Kennedy B, Leblanc Y, Leger S, Mancini J, O'Neill GP, Ouellet M, Percival MD, Perrier H, Riendeau D, Rodger I, Zamboni R (1999) The discovery of rofecoxib, [MK966, Vioxx, 4-(4V-methylsulfonylphenyl)-3-phenyl-2(5H)-furanone], an orally active cyclooxygenase-2 inhibitr. Bioorg Med Chem Lett 9:1773-1778

Ramakrishna V, Gopi S, Setty OH (2011) Indian Gooseberry (Phyllanthus emblica L.) - Phytochemistry, Pharmacology, and Therapeutics. In: Gupta V (ed) Medicinal Plants: Phytochemistry, Pharmacology and Therapeutics Vol. 2. Daya Publishers, New Delhi, pp 19-40

Reddy TC, Aparoy P, Kishore Babu N, Anil Kumar K, Kumar Kalangi S, Reddanna P (2010) Kinetics and Docking Studies of a COX-2 Inhibitor Isolated from Terminalia bellerica Fruits. Protein Pept Lett 17:1251-1257

Sali A, Blundell TL (1993) Comparative protein modeling by satisfaction of spatial restraints. J Mol Biol 234:779-815

Subbaramaiah K, Telang N, Ramonetti JT, Araki R, Devito B, Weksker BB, Dannenberg AJ (1996) Transcription of cyclooxygenase-2 is enhanced in transformed mammary epithelial cells. Cancer Res 56:4424-4429

Subhashini J, Mahipal SVK, Madhava Reddy C, Mallikarjuna Reddy M, Aparna R, Reddanna P (2004) Biochem Pharmacol 68:453

Vane JR, Bakhle YS, Botting RM (1998) Cyclooxygenase 1 and 2. Annu Rev Pharmacol Toxicol 38:97

Verdonk ML, Cole JC, Hartshorn MJC, Murray W, Taylor RD (2003) Improved protein-ligand docking using GOLD. Proteins 52:609-623

Wang Y, Xiao J, Suzek TO, Zhang J, Wang J, Bryant SH (2009) PubChem: a public information system for analyzing bioactivities of small molecules. Nucleic Acids Res 6:1-11 
Wiederstein M, Sippl MJ (2007) ProSA-web: interactive web service for the recognition of errors in threedimensional structures of proteins. Nucleic Acids Res 35:407-410

Willard L, Ranjan A, Zhang H, Monzavi H, Boyko RF, Sykes BD, Wishart DS (2003) VADAR: a web server for quantitative evaluation of protein structure quality. Nucleic Acids Res 31:3316-3319

Zhang F, Altorki NK, Mestre JR, Subbaramaiah K, Dannenberg AJ (1999) Curcumin inhibits cyclooxygenase-2 transcription in bile acid- and phorbol estertreated human gastrointestinal epithelial cells. Carcinogenesis 20(1999):445-451

doi:10.1186/2193-1801-1-58

Cite this article as: Amaravani et al: COX-2 structural analysis and docking studies with gallic acid structural analogues. SpringerPlus 2012 1:58.

Submit your manuscript to a SpringerOpen ${ }^{\circ}$ journal and benefit from:

- Convenient online submission

- Rigorous peer review

- Immediate publication on acceptance

- Open access: articles freely available online

- High visibility within the field

- Retaining the copyright to your article

Submit your next manuscript at $>$ springeropen.com 